\title{
Arabidopsis thaliana Remorins Interact with SnRK1 and Play a Role in Suscepti- bility to Beet Curly Top Virus and Beet Severe Curly Top Virus
}

\author{
Seungmin Son, Chang Jae Oh, and Chung Sun An* \\ School of Biological Sciences, College of Natural Sciences, Seoul National University, Seoul 151-747, Korea
}

(Received on June 17, 2014; Revised on July 22, 2014; Accepted on July 22, 2014)

Remorins, a family of plant-specific proteins containing a variable $\mathbf{N}$-terminal region and conserved $\mathrm{C}$-terminal domain, play a role in various biotic and abiotic stresses, including host-microbe interactions. However, their functions remain to be completely elucidated, especially for the Arabidopsis thaliana remorin group 4 (AtREM4). To elucidate the role of remorins in Arabidopsis, we first showed that AtREM4s have typical molecular characteristics of the remorins, such as induction by various types of biotic and abiotic stresses, localization in plasma membrane and homo- and hetero-oligomeric interaction. Next, we showed that their loss-of-function mutants displayed reduced susceptibility to geminiviruses, Beet Curly Top Virus and Beet Severe Curly Top Virus, while overexpressors enhanced susceptibility. Moreover, we found that they interacted with SnRK1, which phosphorylated AtREM4.1, and were degraded by the $26 \mathrm{~S}$ proteasome pathway. These results suggest that AtREM4s may be involved in the SnRK1-mediated signaling pathway and play a role as positive regulators of the cell cycle during geminivirus infection.

Keywords : Arabidopsis, geminivirus, remorin, SnRK1

Geminiviruses are approximately $2.8 \mathrm{~kb}$ single-stranded circular plant DNA viruses that can cause serious losses of crop products worldwide (Vanderschuren et al., 2007). They are divided into four genera: Mastrevirus, Curtovirus, Topocuvirus, and Begomovirus. Beet curly top virus (BCTV) and Beet severe curly top virus (BSCTV) are Curtoviruses that infect only phloem-limited dicotyledonous plants and raise symptoms such as stunted growth, leaf curling, accumulation of anthocyanin, vein swelling and hyperplasia of the phloem (Latham et al.,

*Corresponding author.

Phone) +82-2-880-6678, FAX) +82-2-872-1993

E-mail) ancs@snu.ac.kr
1997). Geminiviruses regulate several signaling pathways involved in cell cycle regulation and host defense for the purpose of viral propagation. Infection with geminiviruses leads to cell cycle reprogramming using RBR. Viral proteins such as AL1 (also known as AC1, C1 and Rep) and C3 (also known as REn) bind to RBR and inhibit it (Desvoyes et al., 2006; Kong et al., 2000); thus, the infected cells express genes associated with the onset of G1, S and early G2 phases, while suppressing those associated with the early G1 and late G2 phases (Ascencio-Ibanez et al., 2008).

Plants have innate antiviral defense systems such as gene silencing, and regulation of salicylic acid biosynthesis and metabolism (Zhang et al., 2011). Geminiviruses also interfere with these defense systems. To facilitate geminivirus replication, $\mathrm{C} 2$ interacts with $\mathrm{ADK}$ and SAMDC1 and suppresses the plant methyl cycle (Wang et al., 2005; Zhang et al., 2011), and inhibits SnRK1 (Hao et al., 2003). SnRK1-mediated innate antiviral defense was identified by the interaction of geminivirus $\mathrm{C} 2$ proteins with SnRK1.2 (Hao et al., 2003), and it was reported that SlSnRK1 reduces geminivirus infection by interacting with and phosphorylating the $\beta C 1$ protein (Shen et al., 2011). SnRK1 is a key regulator of plant stress and metabolism, and it regulates global transcription (Baena-Gonzalez et al., 2007). Therefore, it was suggested that SnRK1 may also control many levels of transcription during geminivirus infection; however, the antiviral signaling pathway of SnRK1 is largely unknown.

Geminiviruses also protect some unstable host proteins such as GRIK and SAMDC1 from degradation (Shen and Hanley-Bowdoin, 2006; Zhang et al., 2011) and utilize the ubiquitin pathway for viral replication (AlcaideLoridan and Jupin, 2012). They hijack the ubiquitin ligase complexes that are key regulators of several processes, including the cell cycle, for modulating host function. The $\mathrm{C} 2$ protein changes several plant hormone responses using the CUL1-based SCF ubiquitin E3 ligases (LozanoDuran et al., 2011), and C4 activates plant cell proliferation using RKP ligase that targets cyclin kinase inhibitors for 
proteasomal degradation (Lai et al., 2009). In addition, the Clink protein bound to RBR and SKP1 was supposed to alter ubiquitination to affect cell cycle regulation (Aronson et al., 2000). Indeed, during geminivirus infection, there was a general increase in the transcription of genes encoding components of the ubiquitin-proteasome pathway and ubiquitin enzymes (Ascencio-Ibanez et al., 2008).

Remorins are plant-specific proteins, first reported as plasma membrane (PM) proteins in leaves of tomatoes and potatoes phosphorylated in the presence of polygalacturonide (Farmer et al., 1989). Some of them have been found in detergent-insoluble membranes called lipid rafts (Laloi et al., 2007; Lefebvre et al., 2007; Mongrand et al., 2004). Remorins, found in all land plants, are a family of proteins comprised of six different groups (Raffaele et al., 2007). Remorin genes are expressed in diverse tissues, such as embryonic, shoot apex, and vascular tissues (Bariola et al., 2004), and are induced in dehiscent tissues, source parts of the leaves, and aging organs of tobacco (Raffaele et al., 2009b). Furthermore, mRNA and protein levels of some remorins are regulated by various abiotic stressors, hormones and pathogens (Coaker et al., 2004; Li et al., 2012; Nohzadeh Malakshah et al., 2007; Raffaele et al., 2007; Widjaja et al., 2009).

Remorin proteins have a variable N-terminal, a conserved C-terminal (Remorin_C; PF03763), and a Pfam domain. The N-terminal is responsible for structural and functional divergence, whereas the $\mathrm{C}$-terminal, which includes the coiled-coil motif, is important for oligomerization and localization in the PM. Recently, it was shown that the 28-amino acid C-terminal of StREM1.3, the remorin C-terminal Anchor (RemCA), was necessary and sufficient for PM targeting (Perraki et al., 2012).

Studies on plant-virus and plant-microbe interactions have reported a variety of functions for remorin. Researchers have shown that Potato StREM1.3 binds cell wall-derived galacturonides (Reymond et al., 1996) and interacts with the viral protein TGBp1 of potato virus $X$ (Raffaele et al., 2009a); REM1.3 remorin enhances susceptibility to Phytophthora infestans (Bozkurt et al., 2014); Arabidopsis thaliana remorin 1.3 (AtREM1.3) is differentially phosphorylated after treatment with a bacterial elicitor (Benschop et al., 2007; Jarsch and Ott, 2011) and AtREM1.2 has been identified as RIN4, a negative regulator of plant immunity. (Liu et al., 2009). In addition, MtREM2.2, phosphorylated by RLK, regulates bacterial infection (Lefebvre et al., 2010), and AtREM1.3 interacts with IMPa and translocates to the nucleus (Marin et al., 2012). Although remorins have been expected to function as scaffold proteins in many important signaling pathways, their role in the signaling process is remains unknown.

Since there is little information on the remorins belonging to group 4, we studied AtREM4.1 and AtREM4.2 as the first step in understanding their biological functions in Arabidopsis. We found that they are highly regulated by osmotic stress and senescence, and they are positive regulators of geminivirus infection through interacting with SnRK1.

\section{Materials and Methods}

Plant materials and virus inoculation. All Arabidopsis used in this study were of the Columbia (Col-0) ecotype. AtREM4.1-1, AtREM4.2-1 and AtREM4.2-2 mutants were obtained from the SALK (SALK_063269C, SALK_119462 and SALK_143766) and the double mutants were constructed by crossing AtREM4.1 and AtREM4.2 mutants. For the transgenic plants of pAtREM4.1::GUS and pAtREM4.2::GUS, the 1,622 base pair (bp) upstream region of AtREM4.1 and 1,544 bp upstream region of AtREM4.2 were amplified with primers (Supplementary Table 1) by using genomic DNA. These were then cloned into the gateway pBGWFS7 vector. For overexpression lines, AtREM4.1 and AtREM4.2 were inserted into the pEarleyGate102 binary vector (Earley et al., 2006), then transgenic plants were generated using Agrobacterium tumefaciens GV3101 strains containing the constructs by a floral dip method (Zhang et al., 2006).

For virus inoculation, seeds were sowed in soil for 3 days at $4^{\circ} \mathrm{C}$, and plants were grown under $100 \mu \mathrm{mole} / \mathrm{m}^{2} / \mathrm{s}$ white fluorescent lights with a $16 \mathrm{~h}$ light and $8 \mathrm{~h}$ dark cycle at $22^{\circ} \mathrm{C}$. The Agrobacterium strains containing BCTV, $\mathrm{BSCTV}$, and control vector $\mathrm{pMON}$, were inoculated in the crown of the rosette of four-week-old plants using a needle as previously described (Lee et al., 1994).

Sequence analyses. ClustalW2 (http://www.ebi.ac.uk/ Tools/msa/clustalw2/) and Boxshade (http://www. ch.embnet.org/software/BOX_form.html) programs were used to align the amino acid sequences. Conserved motifs of the protein were identified using MEME (http://meme. sdsc.edu/meme/intro.html) and ELM (http://elm.eu.org/) programs. Coiled-coil motifs were predicted using Marcoil (http://bioinf.wehi.edu.au/f-olders/mauro/Marcoil/index. html). Putative phosphorylation site and PEST motifs were predicted using NetPhos 2.0 (http://www.cbs.dtu.dk/services/NetPhos/) and epestfind (http://emboss.bioinformatics. nl/cgi-bin/emboss/epestfind).

Gene expression analysis. For reverse transcription- 
polymerase chain reaction (RT-PCR), $2 \mu \mathrm{g}$ of total RNA isolated from the plants using TRIzol (Invitrogen, USA) was used as a template for cDNA, and it was performed using the gene specific primers (Supplementary Table 1). The Actin-2 gene was used as a loading control for PCR. For promoter activity analysis, the $\mathrm{T} 3$ transgenic plants of pAtREM4.1::GUS and pAtREM4.2::GUS were treated with MES, $100 \mu \mathrm{M} \mathrm{ABA}$ and $150 \mathrm{mM} \mathrm{NaCl}$ at $3 \mathrm{~h}$. For histochemical analysis, plants were incubated at $37^{\circ} \mathrm{C}$ for $4 \mathrm{~h}$ in the GUS staining buffer $(100 \mathrm{mM}$ sodium phosphate (pH 7.0), $10 \mathrm{mM}$ EDTA, $0.5 \mathrm{mM} \mathrm{K}_{3}\left[\mathrm{Fe}(\mathrm{CN})_{6}\right], 0.5 \mathrm{mM}$ $\mathrm{K}_{4}\left[\mathrm{Fe}(\mathrm{CN})_{6}\right], 0.1 \%$ Triton X-100) supplemented with 0.5 $\mathrm{mM}$ X-Gluc. Chlorophyll was cleared as previously described (Jefferson et al., 1987).

Yeast two-hybrid (Y2H) assay. Full length cDNA, such AtREM1.4, AtREM4.1, AtREM4.2 and SnRK1.2, and truncated cDNA, such as AtREM4.1N (amino acid 1-179), AtREM4.1C (amino acid 180-296), AtREM4.2N (amino acid 1-157) and AtREM4.2C (amino acid 158-275) were amplified with the primers (Supplementary Table 1), and the amplified fragments were ligated into the pGAD GH and pGBT9 BS vectors. The constructs were concurrently transformed to the pJ69-4a yeast strain, and the double transformants in pJ69-4a strains were selected as cells grown in the absence of leucine and tryptophan. The selected double transformants were transferred and grown in the absence of adenosine, histidine, leucine, and tryptophan for interaction selection.

Bimolecular fluorescence complementation (BiFC) and localization of fluorescent conjugated proteins. AtREM1.4, AtREM4.1, AtREM4.2 and SnRK1.2 were amplified with the primers (Supplementary Table 1) and inserted into the pSPYNE173 and pSPYCE(MR) vectors (Waadt et al., 2008). The constructs were transformed to GV3101 and BiFC was performed as previously described (Voinnet et al., 2003). The GV3101 strains contained each construct at an $\mathrm{OD}_{600}$ of 0.5 and were coinfiltrated with the p19 strain at an $\mathrm{OD}_{600}$ of 0.3 into 4-week-old tobacco plants ( $N$. benthamiana). Confocal imaging was performed 3-5 days after infiltration with the LSM700 confocal laserscanning microscope (Carl Zeiss, Germany). To analyze the localization of AtREM4.1-CFP and AtREM4.2-CFP, we used 5-day-old seedlings of Arabidopsis overexpressing AtREM4s, and the confocal images were obtained from root.

Expression and purification of recombinant proteins. AtREM4.1, AtREM4.2, GRIK1, and truncated SnRK1.2 (amino acid 1-342) were amplified with the primers listed in Supplementary Table 1. PCR products were cloned into pGEX4T-1 and transformed into Escherichia coli strain BL21(DE3)pLysS. Cells in log-phase growth were grown in the presence of $1 \mathrm{mM}$ IPTG for $20 \mathrm{~h}$ at $20^{\circ} \mathrm{C}$. Total protein was extracted using BugBuster (Novagen, USA) and purified using GST resin (ElpisBiotech, Korea).

In vitro kinase assay. In vitro kinase assay was performed as previously described (Shen et al., 2009). Recombinant proteins $(250 \mathrm{nM})$ were incubated with reaction buffer (25 mM Tris-HCl, pH7.5, 10 mM MgCl 2,1 mM EGTA, 1 mM DTT, $0.2 \mathrm{mM}$ cold ATP, and $0.5 \mu \mathrm{Ci} / \mu \mathrm{L}\left[\gamma^{\left.\left.-{ }^{32} \mathrm{P}\right] \mathrm{ATP}\right)}\right.$ at $30^{\circ} \mathrm{C}$ for $1 \mathrm{~h}$, and the reaction was stopped by $5 \times$ SDSPAGE sample buffer. An equal volume of each sample was loaded and separated in $8 \%$ SDS-PAGE gels. After protein separation, the gels were lapped and ${ }^{32} \mathrm{P}$-labeled proteins were visualized by autoradiography on X-ray films (Fujifilm, Japan).

Cell free degradation assay. Seedling powders of Arabidopsis that were ground in liquid nitrogen were resuspended in degradation buffer containing $25 \mathrm{mM}$ Tris- $\mathrm{HCl}$, pH 7.5, $10 \mathrm{mM} \mathrm{NaCl}, 10 \mathrm{mM} \mathrm{MgCl} 2,5 \mathrm{mM}$ DTT, $10 \mathrm{mM}$ ATP and $4 \mathrm{mM}$ PMSF and total proteins were extracted as previously described (Wang et al., 2009). Recombinant AtREM4 proteins were incubated in equal quantities of extracts with DMSO or $40 \mu \mathrm{M}$ MG132 at $22^{\circ} \mathrm{C}$ for the indicated times, and the reaction was stop by $5 \times$ SDS-PAGE sample buffer. An equal volume of each sample was separated in $8 \%$ SDS-PAGE gel, and immunoblots were performed with GST antibody. The band quantifications of the remaining protein on immunoblots were measured using ImageJ (http://rsb.info.nih.gov/ij/index.html).

\section{Results}

Transcription of AtREM4s is highly enhanced by osmotic stress, abscisic acid and senescence. There are 16 remorin genes in A. thaliana and they are expected to be involved in various biotic and abiotic signaling pathways. However, their function is not known yet. To elucidate the role of remorin, we examined the expression levels of 16 remorin genes of Arabidopsis using the Genevestigator program (https://www.genevestigator.com/gv/) (data not shown). Interestingly, AtREM4.1 and AtREM4.2 were highly regulated by various osmotic stressors, ABA, high light, and pathogens. They have similar sequences and are likely to be recent duplicates of each other (Raffaele et al., 2007). Deduced AtREM4 proteins have proline and serine- 
$\mathbf{A}$

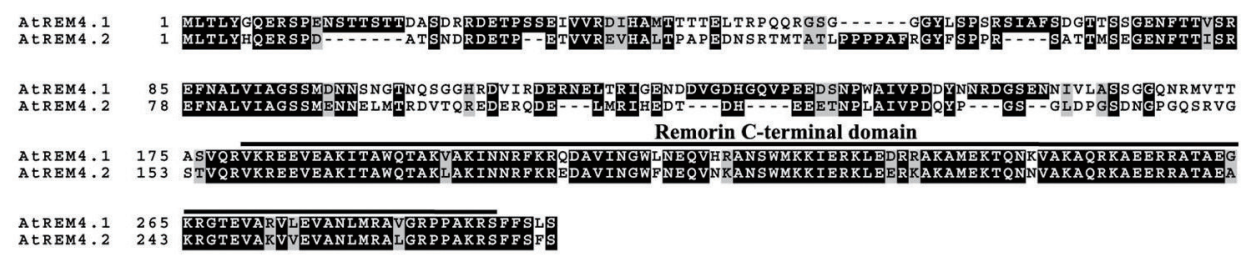

B

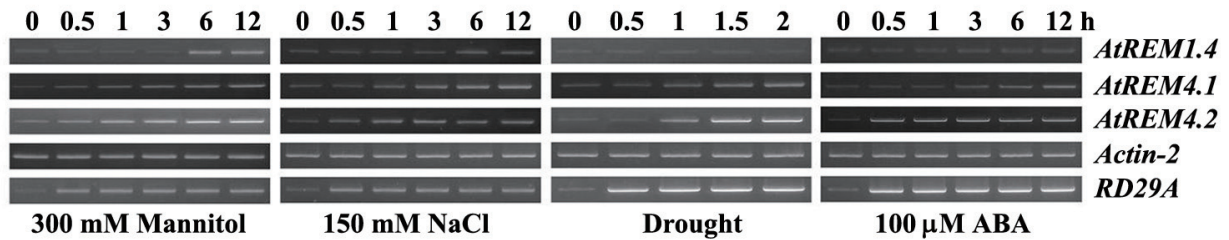

$\mathbf{C}$

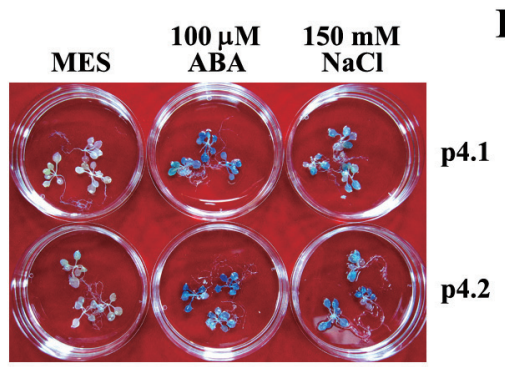

D
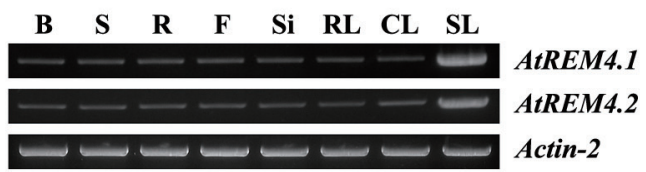

Fig. 1. The amino acid alignment and expression level of AtREM4s. (A) The amino acids sequence alignment of AtREM4s were constructed using the ClustalW software. Black boxes represent conserved amino acids and remorin C-terminal domains that were predicted by the Marcoil software. (B) RT-PCR was performed for stress responses using 2-week-old plants treated with $300 \mathrm{mM}$ mannitol, 150 $\mathrm{mM} \mathrm{NaCl}$, drought and $100 \mu \mathrm{M}$ ABA at indicted time. Actin-2 is used for a loading control and RD29A as a stress marker. (C) Promoter activity for stress response analysis. T3 transgenic plants were treated with MES, $100 \mu \mathrm{M}$ ABA and $150 \mathrm{mM} \mathrm{NaCl}$ at $3 \mathrm{~h}$, and then GUSstaining was performed. The 1,622-bp upstream region of AtREM4.1 is indicated by p4.1, and p4.2 represents the 1,544-bp upstream region of AtREM4.2. (D) RT-PCR analysis for various tissues. Actin-2 was used for loading control. B, bud; S, stem; R, root; F, flower; $\mathrm{Si}$, silique; RL, rosette leaf; CL, cauline leaf; SL, senescence leaf.

rich $\mathrm{N}$-terminal regions and each have the $89 \%$ conserved remorin $\mathrm{C}$-terminal domain, a typical remorin protein structure (Fig. 1A). To validate the expression levels of AtREM4s, we performed RT-PCR using gene specific primers in various osmotic and ABA conditions. Indeed, their expressions were highly enhanced by mannitol, $\mathrm{NaCl}$, drought, and ABA conditions (Fig. 1B). Moreover, their promoters were also activated strongly by $\mathrm{NaCl}$ and $\mathrm{ABA}$ (Fig. 1C). These two genes were predominantly detected in bud, stem, root, flower, silique, and leaves, and enhanced dramatically in senescence leaf (Fig. 1D).

AtREM4 proteins form homo- and hetero-interactions in the plasma membrane. Remorin proteins are reported to localize in the PM, but localization of group 4 remorin proteins remain unknown. Therefore, in order to analyze the localization of AtREM4s in Arabidopsis, we used
35S::AtREM4-CFP transgenic plants. As a result, we could show that AtREM4s also targeted the PM (Fig. 2A). The remorin C-terminal domain, including the coiled-coil motif, was reported to mediate homo-interactions (Toth et al., 2012). Thus, we tested whether AtREM4 proteins that have similar amino acid sequences can interact with each other. In the yeast-two hybrid system, AtREM4s formed homoand hetero-interactions, however, they did not interact with group 1 remorin protein AtREM1.4 (Fig. 2B). To confirm this result in planta, we performed $\mathrm{BiFC}$ using $N$. benthamiana leaves, where strong interactions of AtREM4s were observed in the PM of infiltrated tobacco epidermal cells (Fig. 2C).

Double mutants of AtREM4s reduce the BCTV and BSCTV susceptibility. To study the function of AtREM4s, we generated double mutants (Fig. 3A) and overexpression 
$\mathbf{A}$

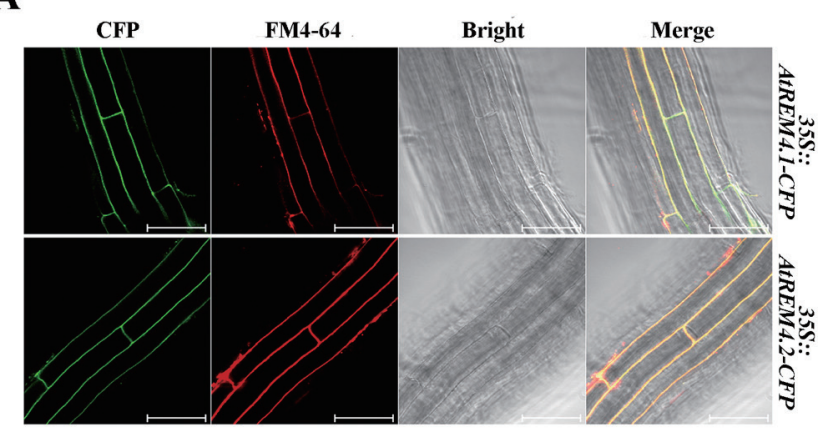

B
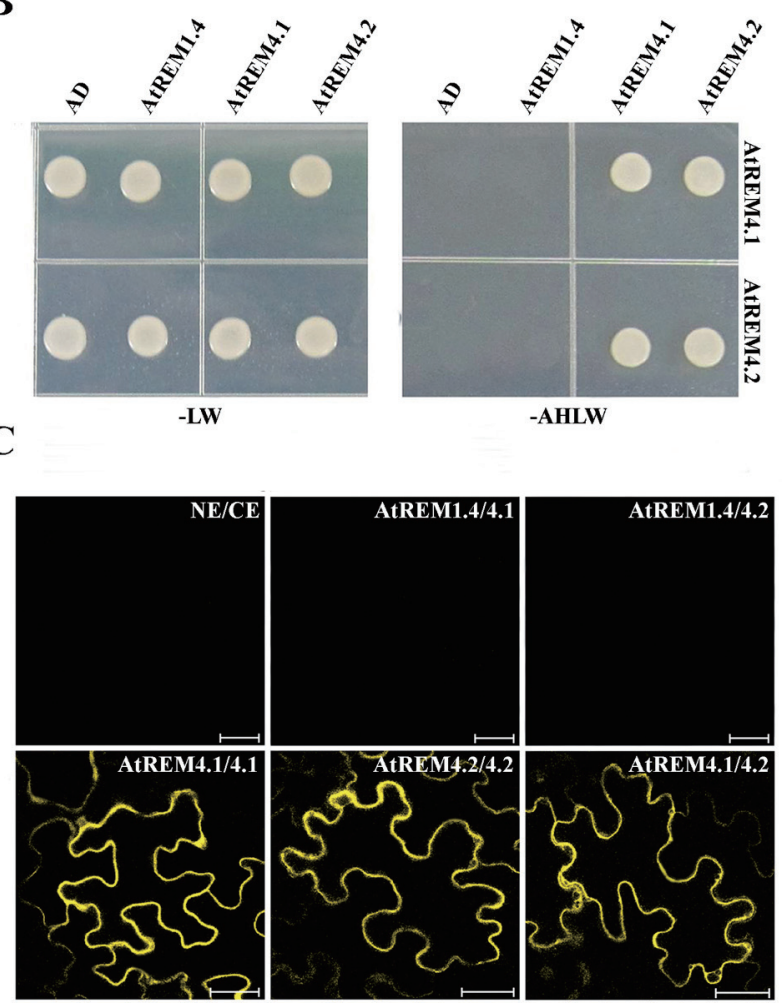

Fig. 2. Subcellular localization and oligomeric interaction of AtREM4s. (A) Confocal images of 35S::AtREM4.1-CFP and 35S::AtREM4.2-CFP in 5-day-old seedlings. FM4-64 was used for PM staining marker. Bars $=50 \mu \mathrm{m}$. (B) $\mathrm{Y} 2 \mathrm{H}$ analysis for interaction among AtREM1.4, AtREM4.1, and AtREM4.2. (C) $\mathrm{BiFC}$ analysis of homo- and hetero-oligomeric interactions between AtREM4s in N. benthamiana, as described in the Materials and Methods. The fluorescence indicates the interaction between the indicated partner proteins. BiFC cloning vectors, $\mathrm{NE}$ and $\mathrm{CE}$, and AtREM1.4 were used as control. Scale bar $=50 \mu \mathrm{m}$.

lines (data not shown) to test their involvement in plantmicrobe interaction. Interestingly, AtREM4 double mutants showed reduced BCTV and BSCTV susceptibility (Fig. 3B). To confirm this, we inoculated the Agrobacterium that contained the BSCTV genome to AtREM4 mutant and overexpression lines. As a result, single mutants showed slightly reduced susceptibility, whereas overexpression lines showed severe stunting of growth, severe curling of leaves and malformed inflorescence structures (Fig. 3C). Based on severity of infection rates, classified as previously described (Park et al., 2011), the difference was more evident (Fig. 3D). At 3 weeks after BSCTV inoculation, approximately $75 \%$ of wild-type (WT) plants developed severe symptoms. However only $10 \%$ of the plant double mutants showed severe symptoms, while more than $80 \%$ of the overexpression lines showed severe infection. Next, RT-PCR was performed to examine the infectivity at molecular level. It was reported that $A T H B 12$ and $R K P$ were induced by BSCTV C4 (Lai et al., 2009; Park et al., 2011). Thus, we used $A T H B 12$ and $R K P$ as marker genes of BSCTV susceptibility. $A T H B 12$ and $R K P$ were expressed highly in WT and overexpression lines, but slightly in double mutants (Fig. 3E). The gene profiling data showed that markers of SA response such as $P R 1$ and $P R 5$ were elevated during geminivirus infection, while transcripts for some JA markers such as $P D F 1.2$ were reduced (AscencioIbanez et al., 2008; Yang et al., 2008). Indeed, PR5 was increased more in WT and overexpression lines than mutant lines, whereas PDF1.2 higher in mutant lines than WT and overexpression lines (Fig. 3E). These results indicate that AtREM4 regulate positively the BCTV and BSCTV susceptibility.

AtREM4s interact with SnRK1.2 and AtREM4.1 is phosphorylated by it. Using computer software, electrophoretic mobility, and heat stability, we found that AtREM4s have intrinsically disordered $\mathrm{N}$-terminal regions (data not shown). They are flexible and modified by several different enzymes such as kinases, ubiquitin-ligases, acetyltransferases and methylases (Dyson and Wright, 2005). Indeed, a bioinformatic analysis of AtREM4s predicted putative phosphorylation sites and PEST motifs within the Nterminal regions (Fig. 4A). Using Y2H library screening, we identified AtREM4-interacting protein partners including SnRK1.2 (data not shown). AtREM4s has many serines and threonines in the N-terminal region, and the phosphorylation prediction program NetPhos 2 predicted more than 20 amino acids to be phosphorylated (Fig. 4A). Thus, we examined which regions mediated this interaction using truncated versions of the gene. N-terminals of AtREM4s interacted with SnRK1.2, but C-terminals did not (Fig. 4B). To address whether AtREM4s are phosphorylated by SnRK1.2, we performed in vitro kinase assays using recombinant proteins with GRIK1, an upregulator of SnRK1 (Hao et al., 2003; Shen et al., 2011). Various combinations of recombinant AtREM4s, SnRK1.2 and GRIK1 proteins 
$\mathbf{A}$

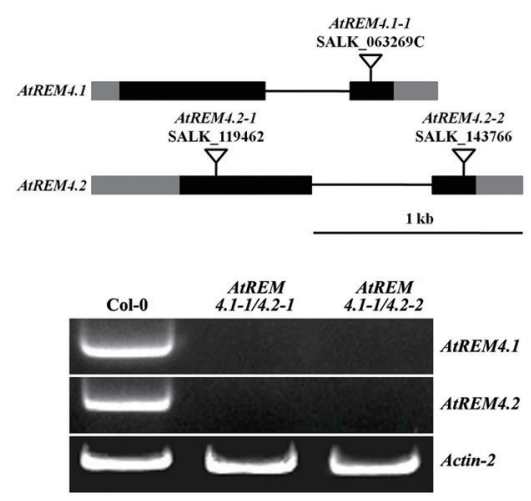

B Mock BCTV BSCTV Mock BCTV BSCTV

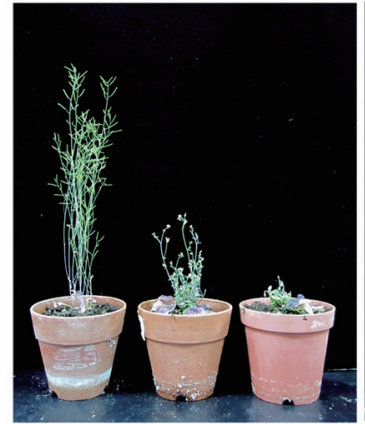

Col-0

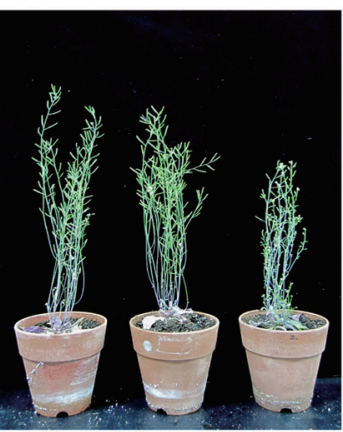

AtREM4.1-1/4.2-1

C

Col-0 AtREM4.1-1 AtREM4.2-1 AtREM4.1-1/4.2-1 AtREM4.1 AtREM4.2

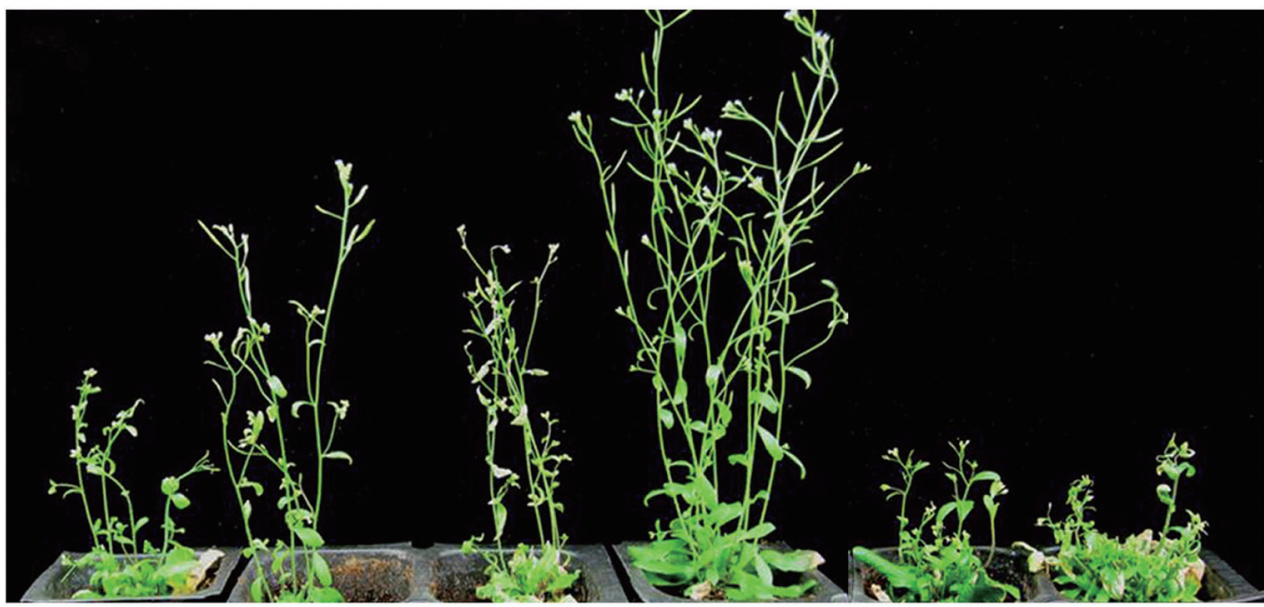

$\mathbf{D}$

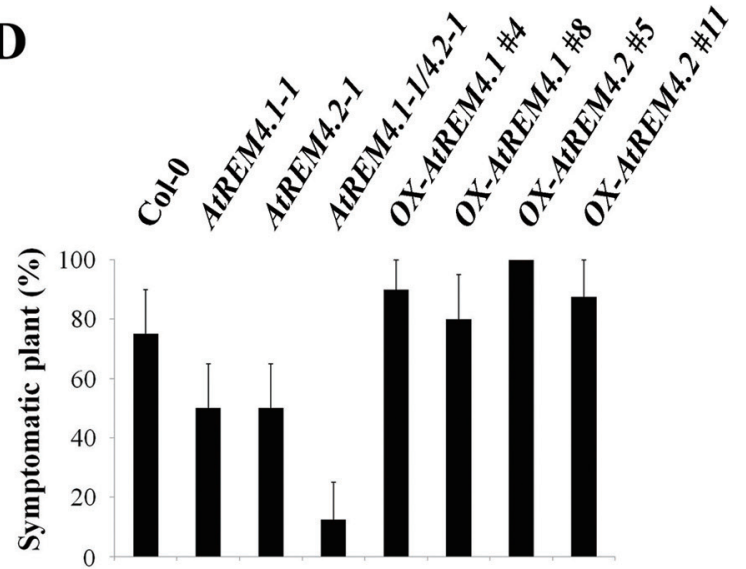

$\mathbf{E}$
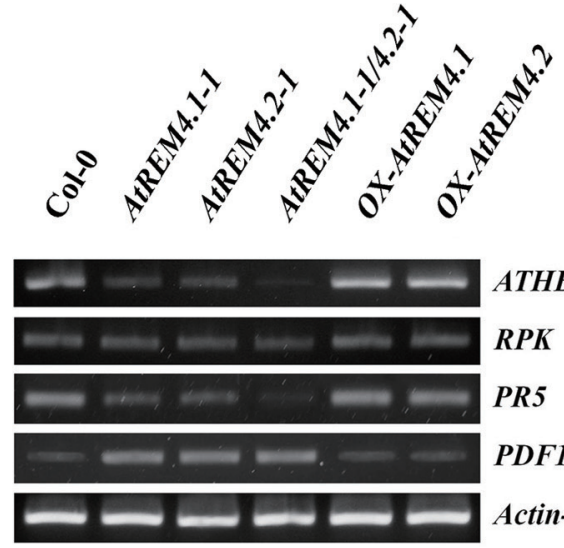

ATHB12

$R P K$

PR5

PDF1.2

Actin-2

Fig. 3. Geminivirus susceptibility of AtREM4s. (A) The gene structures and T-DNA insertion sites. Black boxes are exons, and gray boxes are untranslated regions. Intergenic regions or introns are marked with lines. Expression levels of AtREM4s in the double mutants as assayed by RT-PCR. (B) For the geminivirus infection experiment, Agrobacterium stains containing BCTV, BSCTV and control vector, pMON, were inoculated in the crown of the rosette of four-week-old WT and double mutant plants using a needle. (C) BSCTV infection experiments were performed using WT, AtREM4.1-1, AtREM4.2-1, AtREM4.1-1/4.2-1, 35S::AtREM4.1-CFP and 35S::AtREM4.2-CPF. (D) For severely infected plants, plant symptom severity rates were classified as previously described (Park et al., 2011). (E) For RTPCR, total RNA was isolated from the plants that were infected with BSCTV, and RT-PCR was performed. The Actin-2 gene was used as a loading control for PCR. 
$\mathbf{A}$

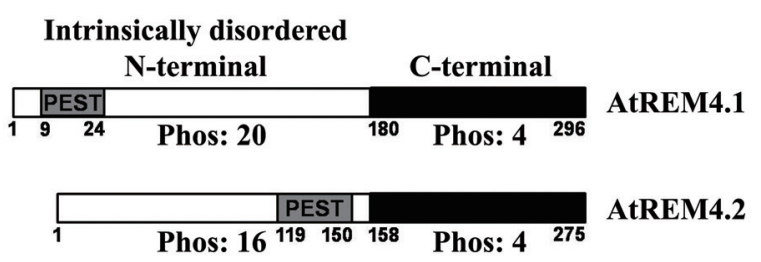

B

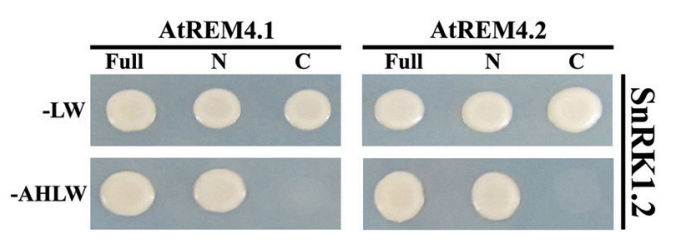

C

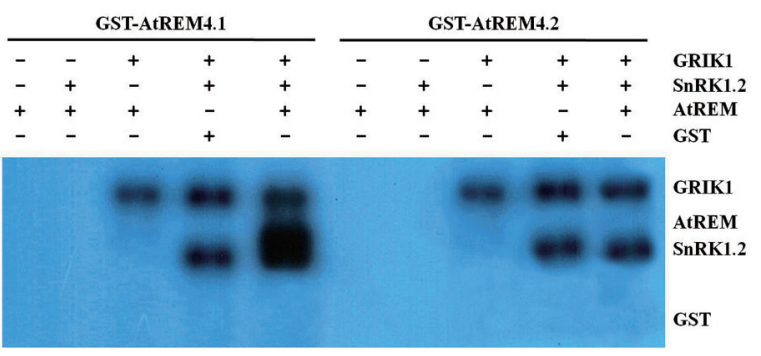

D

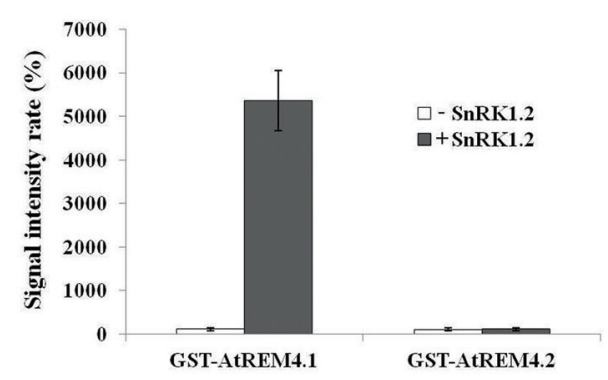

Fig. 4. Phosphorylation of AtREM4s by SnRK1.2 in vitro. (A) Schematic representation of AtREM4 domains. Putative PEST domain and phosphorylation site numbers are shown. Numbers indicate amino acid positions. (B) $\mathrm{Y} 2 \mathrm{H}$ analysis to determine interacting domains of AtREM4s with SnRK1. Constructs of full length and truncated $\mathrm{N}$ and $\mathrm{C}$-terminal ends of AtREM4s were used. (C) In vitro kinase assay for recombinant proteins of AtREM4s, SnRK1.2 and GRIK1. The reactions were resolved by SDS-PAGE gel and visualized by autoradiography. (D) Quantification analysis for the band signal intensity was measured by ImageJ, and the signal intensity rates were calculated such as (GSTAtREM4 intensity/GST intensity) $\times 100$.

were incubated in the presence of $\left[\gamma^{-}{ }^{32} \mathrm{P}\right] \mathrm{ATP}$, and phosphorylation was visualized by autoradiography. The signal band was detected strongly in the GST-AtREM4.1 loading line, but not in the GST-AtREM4.2 line (Fig. 4C). Quanti-
$\mathbf{A}$

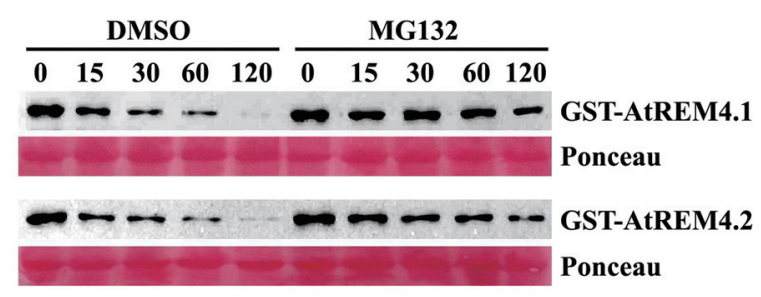

B

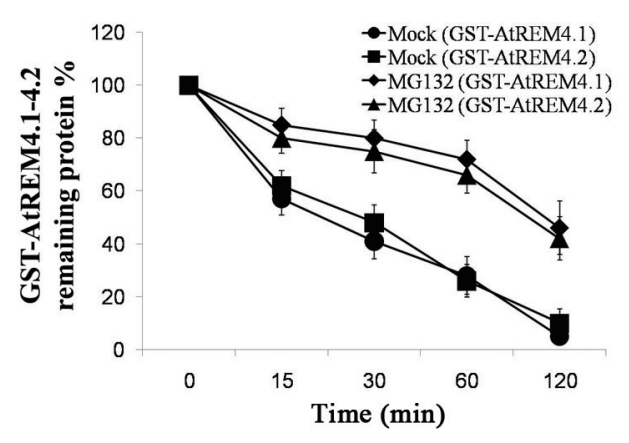

Fig. 5. Cell free degradation assay of recombinant AtREM4 proteins. (A) Immunoblot analysis for GST-AtREM4s degradation in the presence or absence of the proteasome inhibitor MG132. Recombinant GST-fusion proteins were expressed and purified from $E$. coli and then added to the total proteins that were extracted from Col-0 for the indicated times. Immunoblots were probed with anti-GST antibody. Ponceau stain is shown as a loading control. (B) Quantification of the immunoblot analysis was performed using ImageJ.

tative analysis of the signal intensity rate also showed that only GST-AtREM4.1 shows an intense signal (Fig. 4D).

AtREM4s are degraded by the 26S proteasome pathway. Many unstable proteins that are degraded by the $26 \mathrm{~S}$ proteasome have a PEST motif (Rechsteiner and Rogers, 1996; Rogers et al., 1986). Thus, we tested whether AtREM4 proteins are degraded in a proteasome-dependent manner. In the mock, GST-tagged AtREM4s were degraded rapidly, whereas the proteasome inhibitor MG132 apparently delayed the degradation such that with MG132 (Fig. 5A), GST-tagged AtREM4s remained $42-46 \%$ after $2 \mathrm{~h}$, however, in mock, it remained only $5-10 \%$ (Fig. 5B). These results indicated that AtREM4s are degraded by the $26 \mathrm{~S}$ proteasome.

\section{Discussion}

In this study, we present for the first time, the molecular characteristics of AtREM4s and their putative roles during the geminivirus infection process. 
AtREM4s are typical remorins and their expression is highly stimulated by osmotic stress and senescence. To date, most of the studies on remorins have focused on group 1 and 2. Thus, in this study, as the first step in elucidating the biological function of remorin group 4, we studied AtREM4.1 and AtREM4.2. They showed typical characteristics of the remorin molecules, which consist of variable $\mathrm{N}$-terminal and conserved $\mathrm{C}$-terminal regions such as the remorin group 1 proteins (Fig. 1A). Remorins have been known to respond to various stress or developmental conditions. Transcription rates and protein levels of some group 1 remorins were regulated by salt, drought and ABA (Nohzadeh Malakshah et al., 2007; Raffaele et al., 2007), and the transcription of NtREM1.2 increased with organ aging (Raffaele et al., 2009b). AtREM4s were also regulated similarly by abiotic and biotic stressors. However, of the 16 AtREMs, the expression of AtREM4s were induced to the greatest extent by various osmotic stressors and ABA (Fig. 1B). They were also expressed strongly in senescing leaves (Fig. 1D). Moreover, AtREM4s were localized in PM (Fig. 2A) and formed homo-oligomers (Fig. 2B) such as StREM1.3, AtREM1.3 and MtREM2.2. Interestingly, AtREM4s also formed hetero-oligomers, while did not with AtREM1.4 (Fig 2B), suggesting that they could share interacting components each other, and play redundant roles in the same signaling pathway.

AtREM4s enhance susceptibility to geminivirus by mediating SnRK1 and RBR interaction. In this study, we show that AtREM4s enhance susceptibility to geminivirus: overexpressors showed severe infection, while double mutants showed resistance (Fig. 3B). Interestingly, AtREM4s have putative motifs such as the FHA, BRCT and WW domains found in regulating complexes of cell division and DNA damage repair proteins. Geminivirus infection leads to cell cycle reprograming of host plants and activation of G1/S transitioning for geminivirus DNA replication. RBR is a key negative regulator of that transition in plant cells (Gutzat et al., 2012). It binds and inactivates E2F transcription factors required for the expression of genes such as the host replication initiator proteins (Rep). Indeed, interaction of Rep with RBR led to the development of geminivirus symptoms (Kong et al., 2000). Moreover, we show that AtREM4s interacted with SnRK1 (Fig. 4B), by which AtREM4.1 was phosphorylated in vitro (Fig. 4C). GRIK, identified as Rep interacting protein, (Kong and HanleyBowdoin, 2002) phosphorylated SnRK1 (Shen et al., 2009). SnRK1, a key regulator of plant stress and metabolism, is involved in plant antiviral defense. Overexpression of SnRK1 resulted in reduction of geminivirus infection, while silencing of it resulted in the accumulation of more viral DNA than wild-type plants (Hao et al., 2003; Shen et al., 2011). These results suggest that AtREM4s may be one of the intermediate regulators between SnRK1 and RBR during geminivirus infection. Alternatively, they might be involved in a SnRK1-mediated defense-signaling pathway. To prove this hypothesis, we are in the process of elucidating the functional roles of AtREM4 and AtREM4-interacting partners using genetic and cell-biological approaches.

In addition, AtREM4s interacted with some ubiquitinproteasome components (data not shown) and AtREM4.1 was degraded by the $26 \mathrm{~S}$ proteasome (Fig. 5A). Previously, there were some reports that remorin protein levels were increased, while the expression was not changed or even decreased (Nohzadeh Malakshah et al., 2007; Widjaja et al., 2009). When we searched the PEST motifs, known as a signal for protein degradation, among 16 remorin proteins in Arabidopsis, they were predicted in N-terminals of 14 remorins except AtREM3.1 and AtREM3.2 (data not shown). These results suggest that decreased protein levels of the remorins mediated by the $26 \mathrm{~S}$ proteasome, such as degradation of AtREM4 or AtREM4-interacting protein during the geminivirus infection process, might also be an important mechanism in regulating remorin-mediated signaling processes.

\section{Acknowledgments}

The Agrobacterium stains containing BCTV, BSCTV, and control vector pMON were kindly provided by Professor Sukchan Lee (Department of Genetic Engineering, Sungkyunkwan University, Suwon, Korea). This study was supported by a grant from the National Research Foundation, Republic of Korea (Project No. 2010-0013085).

\section{References}

Alcaide-Loridan, C. and Jupin, I. 2012. Ubiquitin and plant viruses, let's play together! Plant Physiol. 160:72-82.

Aronson, M. N., Meyer, A. D., Gyorgyey, J., Katul, L., Vetten, H. J., Gronenborn, B. and Timchenko, T. 2000. Clink, a nanovirus-encoded protein, binds both $\mathrm{pRB}$ and SKP1. J. Virol. 74:2967-2972.

Ascencio-Ibanez, J. T., Sozzani, R., Lee, T. J., Chu, T. M., Wolfinger, R. D., Cella, R. and Hanley-Bowdoin, L. 2008. Global analysis of Arabidopsis gene expression uncovers a complex array of changes impacting pathogen response and cell cycle during geminivirus infection. Plant Physiol. 148:436-454.

Baena-Gonzalez, E., Rolland, F., Thevelein, J. M. and Sheen, J. 2007. A central integrator of transcription networks in plant stress and energy signalling. Nature 448:938-942. 
Bariola, P. A., Retelska, D., Stasiak, A., Kammerer, R. A., Fleming, A., Hijri, M., Frank, S. and Farmer, E. E. 2004. Remorins form a novel family of coiled coil-forming oligomeric and filamentous proteins associated with apical, vascular and embryonic tissues in plants. Plant Mol. Biol. 55:579-594.

Benschop, J. J., Mohammed, S., O’Flaherty, M., Heck, A. J., Slijper, M. and Menke, F. L. 2007. Quantitative phosphoproteomics of early elicitor signaling in Arabidopsis. Mol. Cell Proteomics 6:1198-1214.

Bozkurt, T. O., Richardson, A., Dagdas, Y. F., Mongrand, S., Kamoun, S. and Raffaele, S. 2014. The plant membraneassociated REMORIN1.3 accumulates in discrete perihaustorial domains and enhances susceptibility to Phytophthora infestans. Plant Physiol. 165:1005-1018.

Coaker, G. L., Willard, B., Kinter, M., Stockinger, E. J. and Francis, D. M. 2004. Proteomic analysis of resistance mediated by $\mathrm{Rcm} 2.0$ and $\mathrm{Rcm}$ 5.1, two loci controlling resistance to bacterial canker of tomato. Mol. Plant-Microbe Interact. 17:1019-1028.

Desvoyes, B., Ramirez-Parra, E., Xie, Q., Chua, N. H. and Gutierrez, C. 2006. Cell type-specific role of the retinoblastoma/ E2F pathway during Arabidopsis leaf development. Plant Physiol. 140:67-80.

Dyson, H. J. and Wright, P. E. 2005. Intrinsically unstructured proteins and their functions. Nat. Rev. Mol. Cell Biol. 6:197208.

Earley, K. W., Haag, J. R., Pontes, O., Opper, K., Juehne, T., Song, K. and Pikaard, C. S. 2006. Gateway-compatible vectors for plant functional genomics and proteomics. Plant J. 45:616-629.

Farmer, E. E., Pearce, G. and Ryan, C. A. 1989. In vitro phosphorylation of plant plasma membrane proteins in response to the proteinase inhibitor inducing factor. Proc. Natl. Acad. Sci. USA 86:1539-1542.

Gutzat, R., Borghi, L. and Gruissem, W. 2012. Emerging roles of RETINOBLASTOMA-RELATED proteins in evolution and plant development. Trends Plant Sci. 17:139-148.

Hao, L., Wang, H., Sunter, G. and Bisaro, D.M. 2003. Geminivirus AL2 and L2 proteins interact with and inactivate SNF1 kinase. Plant Cell 15:1034-1048.

Jarsch, I. K. and Ott, T. 2011. Perspectives on remorin proteins, membrane rafts, and their role during plant-microbe interactions. Mol. Plant-Microbe Interact. 24:7-12.

Jefferson, R. A., Kavanagh, T. A. and Bevan, M. W. 1987. GUS fusions: beta-glucuronidase as a sensitive and versatile gene fusion marker in higher plants. EMBO J. 6:3901-3907.

Kong, L. J. and Hanley-Bowdoin, L. 2002. A geminivirus replication protein interacts with a protein kinase and a motor protein that display different expression patterns during plant development and infection. Plant Cell 14:1817-1832.

Kong, L. J., Orozco, B. M., Roe, J. L., Nagar, S., Ou, S., Feiler, H. S., Durfee, T., Miller, A. B., Gruissem, W., Robertson, D. and Hanley-Bowdoin, L. 2000. A geminivirus replication protein interacts with the retinoblastoma protein through a novel do- main to determine symptoms and tissue specificity of infection in plants. EMBO J. 19:3485-3495.

Lai, J., Chen, H., Teng, K., Zhao, Q., Zhang, Z., Li, Y., Liang, L., Xia, R., Wu, Y., Guo, H. and Xie, Q. 2009. RKP, a RING finger E3 ligase induced by BSCTV C4 protein, affects geminivirus infection by regulation of the plant cell cycle. Plant $J$. 57:905-917.

Laloi, M., Perret, A. M., Chatre, L., Melser, S., Cantrel, C., Vaultier, M. N., Zachowski, A., Bathany, K., Schmitter, J. M., Vallet, M., Lessire, R., Hartmann, M. A. and Moreau, P. 2007. Insights into the role of specific lipids in the formation and delivery of lipid microdomains to the plasma membrane of plant cells. Plant Physiol. 143:461-472.

Latham, J. R., Saunders, K., Pinner, M. S. and Stanley, J. 1997. Induction of plant cell division by beet curly top virus gene C4. Plant J. 11:1273-1283.

Lee, S., Stenger, D. C., Bisaro, D. M. and Davis, K. R. 1994. Identification of loci in Arabidopsis that confer resistance to geminivirus infection. Plant J. 6:525-535.

Lefebvre, B., Furt, F., Hartmann, M. A., Michaelson, L. V., Carde, J. P., Sargueil-Boiron, F., Rossignol, M., Napier, J. A., Cullimore, J., Bessoule, J. J. and Mongrand, S. 2007. Characterization of lipid rafts from Medicago truncatula root plasma membranes: a proteomic study reveals the presence of a raftassociated redox system. Plant Physiol. 144:402-418.

Lefebvre, B., Timmers, T., Mbengue, M., Moreau, S., Herve, C., Toth, K., Bittencourt-Silvestre, J., Klaus, D., Deslandes, L., Godiard, L., Murray, J. D., Udvardi, M. K., Raffaele, S., Mongrand, S., Cullimore, J., Gamas, P., Niebel, A. and Ott, T. 2010. A remorin protein interacts with symbiotic receptors and regulates bacterial infection. Proc. Natl. Acad. Sci. USA 107:2343-2348.

Li, B., Zhang, C., Cao, B., Qin, G., Wang, W. and Tian, S. 2012. Brassinolide enhances cold stress tolerance of fruit by regulating plasma membrane proteins and lipids. Amino Acids 43:2469-2480.

Liu, J., Elmore, J. M., Fuglsang, A. T., Palmgren, M. G., Staskawicz, B. J. and Coaker, G. 2009. RIN4 functions with plasma membrane $\mathrm{H}^{+}$-ATPases to regulate stomatal apertures during pathogen attack. PLoS Biol. 7:e1000139.

Lozano-Duran, R., Rosas-Diaz, T., Gusmaroli, G., Luna, A. P., Taconnat, L., Deng, X. W. and Bejarano, E. R. 2011. Geminiviruses subvert ubiquitination by altering CSN-mediated derubylation of SCF E3 ligase complexes and inhibit jasmonate signaling in Arabidopsis thaliana. Plant Cell 23:1014-1032.

Marin, M., Thallmair, V. and Ott, T. 2012. The intrinsically disordered N-terminal region of AtREM1.3 remorin protein mediates protein-protein interactions. J. Biol. Chem. 287:3998239991.

Mongrand, S., Morel, J., Laroche, J., Claverol, S., Carde, J. P., Hartmann, M. A., Bonneu, M., Simon-Plas, F., Lessire, R. and Bessoule, J. J. 2004. Lipid rafts in higher plant cells: purification and characterization of Triton X-100-insoluble microdomains from tobacco plasma membrane. J. Biol. Chem. 
279:36277-36286.

Nohzadeh Malakshah, S., Habibi Rezaei, M., Heidari, M. and Salekdeh, G. H. 2007. Proteomics reveals new salt responsive proteins associated with rice plasma membrane. Biosci. Biotechnol. Biochem. 71:2144-2154.

Park, J., Lee, H. J., Cheon, C. I., Kim, S. H., Hur, Y. S., Auh, C. K., Im, K. H., Yun, D. J., Lee, S. and Davis, K. R. 2011. The Arabidopsis thaliana homeobox gene ATHB12 is involved in symptom development caused by geminivirus infection. PLOS ONE 6:e20054.

Perraki, A., Cacas, J. L., Crowet, J. M., Lins, L., Castroviejo, M., German-Retana, S., Mongrand, S. and Raffaele, S. 2012. Plasma membrane localization of Solanum tuberosum remorin from group 1, homolog 3 is mediated by conformational changes in a novel C-terminal anchor and required for the restriction of Potato Virus $X$ movement. Plant Physiol. 160:624-637.

Raffaele, S., Bayer, E., Lafarge, D., Cluzet, S., German Retana, S., Boubekeur, T., Leborgne-Castel, N., Carde, J. P., Lherminier, J., Noirot, E., Satiat-Jeunemaitre, B., Laroche-Traineau, J., Moreau, P., Ott, T., Maule, A. J., Reymond, P., Simon-Plas, F., Farmer, E. E., Bessoule, J. J. and Mongrand, S. 2009a. Remorin, a solanaceae protein resident in membrane rafts and plasmodesmata, impairs Potato virus $X$ movement. Plant Cell 21:1541-1555.

Raffaele, S., Bayer, E. and Mongrand, S. 2009b. Upregulation of the plant protein remorin correlates with dehiscence and cell maturation: a link with the maturation of plasmodesmata? Plant Signal. Behav. 4:915-919.

Raffaele, S., Mongrand, S., Gamas, P., Niebel, A. and Ott. T. 2007. Genome-wide annotation of remorins, a plant-specific protein family: evolutionary and functional perspectives. Plant Physiol. 145:593-600.

Rechsteiner, M. and Rogers, S. W. 1996. PEST sequences and regulation by proteolysis. Trends Biochem. Sci. 21:267-271.

Reymond, P., Kunz, B., Paul-Pletzer, K., Grimm, R., Eckerskorn, C. and Farmer, E. E. 1996. Cloning of a cDNA encoding a plasma membrane-associated, uronide binding phosphoprotein with physical properties similar to viral movement proteins. Plant Cell 8:2265-2276.

Rogers, S., Wells, R. and Rechsteiner, M. 1986. Amino acid sequences common to rapidly degraded proteins: the PEST hypothesis. Science 234:364-368.

Shen, Q., Liu, Z., Song, F., Xie, Q., Hanley-Bowdoin, L. and Zhou, X. 2011. Tomato SISnRK1 protein interacts with and phosphorylates betaC1, a pathogenesis protein encoded by a geminivirus beta-satellite. Plant Physiol. 157:1394-1406.

Shen, W. and Hanley-Bowdoin, L. 2006. Geminivirus infection up-regulates the expression of two Arabidopsis protein kinas- es related to yeast SNF1- and mammalian AMPK-activating kinases. Plant Physiol. 142:1642-1655.

Shen, W., Reyes, M. I. and Hanley-Bowdoin, L. 2009. Arabidopsis protein kinases GRIK1 and GRIK2 specifically activate SnRK1 by phosphorylating its activation loop. Plant Physiol. 150:996-1005.

Toth, K., Stratil, T. F., Madsen, E. B., Ye, J., Popp, C., AntolinLlovera, M., Grossmann, C., Jensen, O. N., Schussler, A., Parniske, M. and Ott, T. 2012. Functional domain analysis of the Remorin protein LjSYMREM1 in Lotus japonicus. PLoS One 7:e30817.

Vanderschuren, H., Stupak, M., Futterer, J., Gruissem, W. and Zhang, P. 2007. Engineering resistance to geminiviruses-review and perspectives. Plant Biotechnol. J. 5:207-220.

Voinnet, O., Rivas, S., Mestre, P. and Baulcombe, D. 2003. An enhanced transient expression system in plants based on suppression of gene silencing by the 19 protein of tomato bushy stunt virus. Plant J. 33:949-956.

Waadt, R., Schmidt, L. K., Lohse, M., Hashimoto, K., Bock, R. and Kudla, J. 2008. Multicolor bimolecular fluorescence complementation reveals simultaneous formation of alternative CBL/CIPK complexes in planta. Plant J. 56:505-516.

Wang, F., Zhu, D., Huang, X., Li, S., Gong, Y., Yao, Q., Fu, X., Fan, L. M. and Deng, X. W. 2009. Biochemical insights on degradation of Arabidopsis DELLA proteins gained from a cell-free assay system. Plant Cell 21:2378-2390.

Wang, H., Buckley, K. J., Yang, X., Buchmann, R. C. and Bisaro, D. M. 2005. Adenosine kinase inhibition and suppression of RNA silencing by geminivirus AL2 and L2 proteins. J. Virol. 79:7410-7418.

Widjaja, I., Naumann, K., Roth, U., Wolf, N., Mackey, D., Dangl, J. L., Scheel, D. and Lee, J. 2009. Combining subproteome enrichment and Rubisco depletion enables identification of low abundance proteins differentially regulated during plant defense. Proteomics 9:138-147.

Yang, J. Y., Iwasaki, M., Machida, C., Machida, Y., Zhou, X. and Chua, N. H. 2008. betaC1, the pathogenicity factor of TYL$\mathrm{CCNV}$, interacts with AS1 to alter leaf development and suppress selective jasmonic acid responses. Genes Dev. 22:25642577.

Zhang, X. R., Henriques, R., Lin, S. S., Niu, Q. W. and Chua, N. H. 2006. Agrobacterium-mediated transformation of Arabidopsis thaliana using the floral dip method. Nat. Protoc. 1:641-646.

Zhang, Z., Chen, H., Huang, X., Xia, R., Zhao, Q., Lai, J., Teng, K., Li, Y., Liang, L., Du, Q., Zhou, X., Guo, H. and Xie, Q. 2011. BSCTV C2 attenuates the degradation of SAMDC1 to suppress DNA methylation-mediated gene silencing in Arabidopsis. Plant Cell 23:273-288. 